\title{
Supporting Information: A Synthetic DNA Walker for Molecular Transport
}

\author{
Jong-Shik Shin ${ }^{\dagger}$ and Niles A. Pierce ${ }^{*, \dagger}$ \\ ${ }^{\dagger}$ Department of Bioengineering and ${ }^{*}$ Department of Applied \& Computational Mathematics, \\ California Institute of Technology, Pasadena, CA 91125 \\ E-mail:niles@caltech.edu
}

\section{Materials and methods}

Oligonucleotide and Device Preparation. All DNA oligonucleotides were synthesized by Integrated DNA Technologies, Inc. DNA labelling with fluorophore or quencher and subsequent purification were performed by the supplier and the resulting oligomers were used without further purification. DNA stock solutions (40-60 $\mu \mathrm{M})$ were prepared in TE buffer $(\mathrm{pH} \mathrm{8.0)}$ and concentrations were determined at $260 \mathrm{~nm}$ using the molecular extinction coefficient supplied by the manufacturer. The track (final concentration $=5$ $\mu \mathrm{M})$ was prepared by mixing the six track strand species in TSE buffer (10 mM Tris, $150 \mathrm{mM} \mathrm{NaCl}, 1 \mathrm{mM}$ EDTA, pH 7.5), followed by incubation for $3 \mathrm{hr}$ at $37{ }^{\circ} \mathrm{C}$. The walker was prepared by the same procedure using the two walker strand species.

Non-denaturing PAGE Analysis. Fuel-mediated association of the walker and track was analyzed by non-denaturing PAGE. The reaction volume for the PAGE experiments was $10 \mu \mathrm{l}$. Initial anchoring of the walker on branch 1 was achieved by adding equimolar A1 to a reaction mixture of track and walker ( $1 \mu \mathrm{M}$ in TSE buffer), followed by $1 \mathrm{hr}$ incubation at $37^{\circ} \mathrm{C}$. Subsequent walker movement was carried out by successively adding different fuel species in equimolar amounts. Each addition was followed by $1 \mathrm{hr}$ incubation at $37^{\circ} \mathrm{C}$. The whole reaction sample was loaded in a $6.7 \%$ non-denaturing gel. Gel electrophoresis was accomplished in $1 \times \mathrm{TBE}$ buffer at 50 $\mathrm{V}$ and $4{ }^{\circ} \mathrm{C}$, and the bands were visualized by fluorescent scanning (Molecular Imager FX Pro Plus, Bio-Rad).

Multiplexed Real-time Fluorescence Measurement. Fluorescence measurements were carried out with a fluorometer (PTI Co.) at room temperature. Bandwidths for excitation and emission were set to $4 \mathrm{~nm}$ and the working volume for measurements was $100 \mu \mathrm{l}$. Track $(0.5 \mu \mathrm{M})$ was preincubated with equimolar walker and $\mathrm{A} 1$ in
TSE buffer for $4 \mathrm{hr}$ at room temperature. Equimolar amounts of fuel strands were successively added from $100 \times$ stocks and the solution was mixed by rapid pipetting. Fluorescence signals from four different dyes were collected during the same run using the multi-dye mode (Felix32 software, PTI Co.) to monitor fluorescence intensities at four excitation/emission wavelengths.

\section{Independence of fluorescence signals}

To monitor walker movement, four fluorescent dyes were selected (FAM, HEX, Texas Red, Cy5 in order of increasing excitation/emission wavelengths) from many possible candidates. To minimize FRET between dyes, branches 1-4 on the track were labeled in the order (HEX, Cy5, FAM, Texas Red) to double the distance between dyes with adjacent fluorescence spectra. These concerns were particularly relevant because the equilibrium distance between neighboring dyes $(5 \mathrm{~nm})$ is typical of the Förster radius for many dye pairs. Excitation and emission wavelengths for each dye were chosen so as to minimize the response of the other three dyes. Columns 2-5 of Table S1 list fluorescence intensities of the four dye-labled $\mathrm{T}$ strands at the four excitation/emission wavelengths employed for multiplexed fluorescence measurements. At each wavelength, one dye fluoresces strongly and the fluorescence of the other dyes is negligible by comparison ( $1 \%$ or less). Column 6 shows that intensities for the 4-dye full track are within $10 \%$ of those observed for the dominant single strand dye at each excitation/emission wavelength. In addition to possible FRET between dyes, these differences may also result from changes in the local structural environments of the dyes, which often alter fluorescence emissions (even in the absence of a dye pair). Column 7 records the raw initial fluorescence intensities used to normalize the real-time multiplexed fluorescence data of Figure 3.

Table S1. Fluorescence intensities of dye-labled track strands at different wavelengths.

\begin{tabular}{|c|c|c|c|c|c|c|}
\hline Ex/Em (nm) & $\mathrm{HEX}-\mathrm{T} 1^{a, b}$ & $\mathrm{~T} 2-\mathrm{Cy} 5^{a, b}$ & FAM-T3 $^{a, b}$ & T4-Texas $\operatorname{Red}^{a, b}$ & 4-dye Full Track ${ }^{a, b}$ & Fig. 3 initial values \\
\hline 495/520 for FAM & $23,248(0.01)$ & $5,148(0.00)$ & $3,636,790(1.00)$ & $647(0.00)$ & $3,290,060(0.90)$ & $3,318,240$ \\
\hline 538/555 for HEX & $1,690,730(1.00)$ & $3,650(0.00)$ & $4,076(0.00)$ & $844(0.00)$ & $1,527,750(0.90)$ & $1,546,000$ \\
\hline $598 / 617$ for Texas Red & $162(0.00)$ & $1,593(0.00)$ & $105(0.00)$ & $741,071(1.00)$ & $759,613(1.03)$ & 770,900 \\
\hline 648/668 for Сy 5 & $24(0.00)$ & $124,220(1.00)$ & $22(0.00)$ & $126(0.00)$ & $130,448(1.05)$ & 127,131 \\
\hline
\end{tabular}

${ }_{b}^{a}$ Fluorescence intensity (cps) measured at room temperature with samples $(0.5 \mu \mathrm{M})$ in TSE buffer.

${ }^{b}$ Values in parentheses are normalized intensities at a given wavelength (compare within row). 